Artigo / Article

\title{
Contribuição da citometria de fluxo para o diagnóstico e prognóstico das síndromes mielodisplásicas
}

\author{
The application of flow cytometric analysis of bone marrow cells for the diagnosis and \\ prognosis of myelodysplastic syndromes
}

Irene Lorand-Metze

\begin{abstract}
O diagnóstico das síndromes mielodisplásicas (SMD) é baseado nos achados de citopenias no sangue periférico, na morfologia (atipias) das células hemopoiéticas na medula óssea e no cariótipo. Em uma proporção considerável de casos, porém, o grau de atipias encontrado é discreto e sujeito a interpretações subjetivas. Além disso, alterações citogenéticas são encontradas apenas em 30\%-80\% dos casos. A citometria de fluxo multiparamétrica é uma técnica rápida, reproduzível e relativamente barata, capaz de objetivar alterações funcionais do clone SMD na maioria dos casos, o que permite o diagnóstico diferencial com patologias não-clonais que cursam com citopenias periféricas. Várias alterações têm sido descritas na expressão de antígenos ligados a linhagem e maturação celular nas três séries hemopoiéticas. Protocolos de três ou quatro cores analisando-se as séries eritroblástica, mielomonocítica e blastos têm sido propostos e conseguem resolver o diagnóstico diferencial em praticamente todos os casos. A citometria de fluxo também é útil para o acompanhamento dos pacientes, já que a progressão do clone neoplásico é acompanhada por um aumento do número de alterações fenotípicas e de células CD34+ além da diminuição de marcadores pró-apoptóticos. Rev. bras. hematol. hemoter. 2006;28(3):178-181.
\end{abstract}

Palavras-chave: Mielodisplasia; citometria de fluxo; diagnóstico; maturação celular; expressão antigênica.

O diagnóstico das síndromes mielodisplásicas (SMDs) é baseada nos achados do hemograma, citologia e histologia de medula óssea e no cariótipo. ${ }^{1,2}$ Estes achados são suficientes para um diagnóstico inequívoco numa grande proporção de casos. Porém, há casos de citopenias periféricas em que as atipias celulares na medula óssea (MO) não são exuberantes e o cariótipo é normal, ou não revelou mitoses. Nestes casos pode ser difícil o diagnóstico diferencial com patologias não-clonais cursando com citopenias periféricas. $\mathrm{O}$ achado de uma anormalidade citogenética seria a prova mais importante da presença de um distúrbio clonal. Só que isto ocorre apenas em 30\%-80\% dos casos. Para o diagnóstico definitivo destes casos, tem-se lançado mão de um pro- tocolo clínico para exclusão de distúrbios não clonais que cursam com citopenias periféricas, MO celular com algumas atipias. ${ }^{1,3,4}$

Neste contexto, a "imunofenotipagem" tem sido usada no sentido de: (1) detectar expressões anormais de antígenos celulares relacionados a linhagem e maturação das diversas séries hemopoiéticas; (2) detectar aumento de células CD34+ (correlatos dos blastos no mielograma); (3) verificar aumento de marcadores pró-apoptóticos ou mesmo células em apoptose na MO. ${ }^{5-15}$

A citometria de fluxo vem sendo usada para analisar células do sangue e MO nos casos de SMD desde há 15 anos. Inicialmente se usaram relativamente poucos anti-

Professora titular da Disciplina Médica de Hematologia da Faculdade Estadual de Campinas.

Correspondência: Irene Lorand-Metze

Faculdade de Ciências Médicas, Universidade Estadual de Campinas

Hemocentro Unicamp, Caixa Postal 6198

13081-970 - Campinas-SP

ilmetze@unicamp.br 
corpos monoclonais em marcação única ou de duas cores. ${ }^{9}$ O desenvolvimento dos citômetros de dois lasers que permitem uma análise multiparamétrica, estudando-se simultaneamente seis ou mais parâmetros na mesma célula, permitiu o conhecimento da expressão destes antígenos nas diversas linhagens e no processo de maturação celular. Hoje se conhece bem o perfil delas nas diversas linhagens da hemopoiese normal e patológica. ${ }^{9} 16$ Finalmente, abandonou-se a análise de células positivas e negativas para um dado antígeno com pontos de corte para "positivo" e "negativo". Atualmente se quantifica o número de células positivas para cada antígeno (qualquer porcentagem) e se pode medir a intensidade de fluorescência (IMF) que é proporcional à intensidade de expressão de cada antígeno. Porém, o fim principal da análise multiparamétrica é analisar o perfil de expressão de cada subpopulação presente numa amostra de sangue, medula óssea ou outro material biológico. Além do mais, pode-se quantificar o número de células com determinado perfil antigênico. Por ex. blastos CD34+/CD+38/CD33-. ${ }^{10-12}$

Hoje é possível fazer-se um "mielograma" por citometria (Figura 1) se usarmos o anticorpo anti-CD45 e o SSC (dispersão a 90 graus - o que mede a complexidade interna da célula), separando-se assim cada linhagem, que pode ser estudada individualmente quanto ao seu perfil de expressão antigênica.

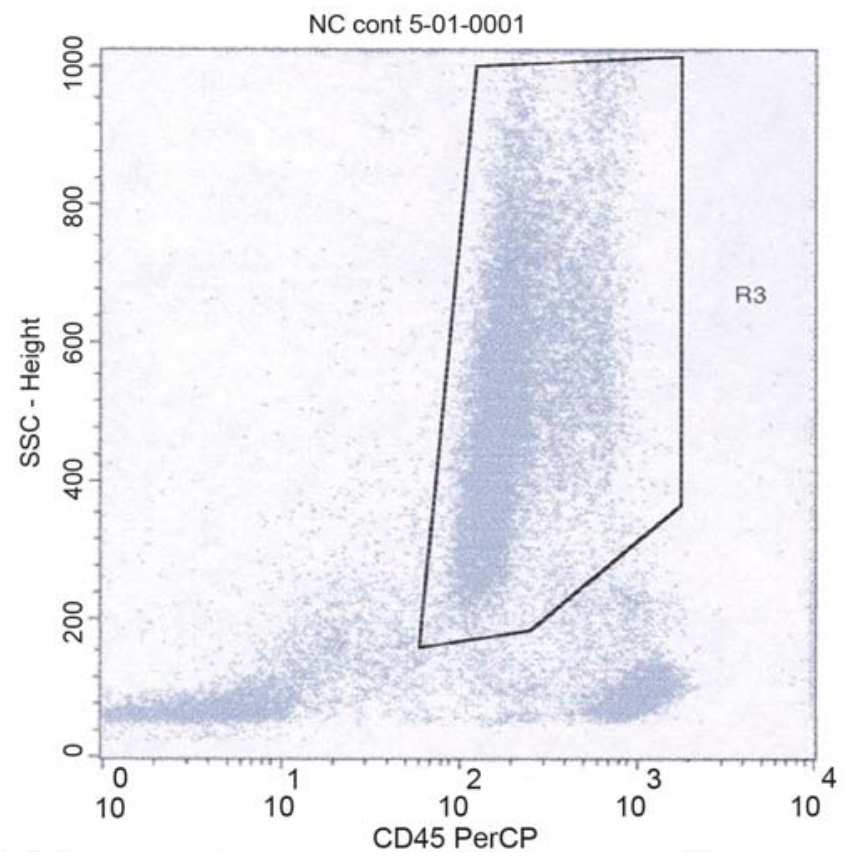

Figura 1. Dot plot CD45/SSC de uma medula óssea normal. Os eritroblastos são CD45 negativos e SSC baixo (população à direita). Os precursores granulocíticos têm expressão de CD45 e SSC variável (R3). Os blastos são encontrados abaixo desta população. À esquerda, embaixo (CD45 fortemente positivo), estão os linfócitos. A população monocítica se localiza entre os linfócitos e monócitos
Durante estes 15 anos do desenvolvimento da citometria de fluxo no estudo das SMDs foram publicados um grande número de trabalhos..$^{5-15}$ Mas cada um deles usou uma abordagem diferente, usando protocolos de duas a quatro cores (ou mais). Desta forma, estudaram-se anormalidades de maturação, ${ }^{2-6}$ número e expressões aberrantes nos blastos $^{5,10-12}$ e o aumento de proliferação e apoptose nas células hemopoiéticas. ${ }^{13,14}$

Para o estudo da linhagem granulocítica, é importante a análise da expressão de CD33, CD13, CD11b, CD117, CD15, CD16 e CD10. Tem-se descrito perda parcial ou total de cada um deles durante a maturação, além de assincronismos de maturação. ${ }^{5-10,16}$ A "hipogranularidade", vista no exame citológico, pode ser objetivada medindo-se o IMF do SSC para toda a população granulocítica ou para um determinado tipo de célula (ex. células CD34+).

Além disso, conhecem-se as características fenotípicas do desvio à esquerda durante a recuperação da granulopoese normal após quimioterapia ou o uso de fatores de crescimento: expressão anormalmente homogênea e blastos de HLA-DR, CD33, CD34 e CD38; aumento do número de células na transição entre o gate de blastos e granulócitos (Figura 1); além do aumento do número de células com expressão fraca de CD16 e CD11b e alta expressão de CD33. ${ }^{9}$

São consideradas alterações específicas de SMD: (1) perda parcial ou total de um antígeno em pelo menos $10 \%$ das células em uma população de interesse; (2) parada de maturação (expressão homogênea, sem a variação maturacional normal (ex. CD13); (3) expressão assíncrona de dois antígenos durante a maturação; (4) expressões aberrantes: presença de CD5, CD7 ou CD56 (em mais de 10\% das células) na linhagem granulocítica (este achado é pouco freqüente); (5) SSC diminuído nos granulócitos (hipogranularidade). ${ }^{9,16-18}$

Os monócitos também têm sido estudados. É descrito um aumento do seu número bem como perda parcial ou total de CD13, CD14 ou CD15., ${ }^{9,16-18}$

A série eritroblástica é geralmente analisada pela expressão da glicoforina A e do CD71 (receptor de transferrina). Embora vários autores tenham descrito também alterações nos megacariócitos e plaquetas, ${ }^{5,6}$ estes são mais difíceis de analisar, pelo seu tamanho, pequeno número e dificuldades técnicas.

De modo geral, há boa correlação entre o número de células CD34+ na citometria de fluxo e a porcentagem de blastos contados no mielograma. ${ }^{4,5,14}$ Nestas células tem-se descrito o fenótipo CD34+/CD38+/CD33+ bem como assincronismos de maturação, mas raramente co-expressões anômalas. ${ }^{710-12}$ Além disso, encontramos hiperexpressão de Fas (CD95) e FasL nas SMDs de baixo risco. ${ }^{13,14}$ Esta expressão cai à medida que aumenta o número de blastos. Todas estas alterações acima descritas são muito freqüentes (70\%-88\%) e o seu tipo e número correlaciona com diversas alterações 
citogenéticas, ${ }^{7-9}$ com o IPSS bem como com o recém-descrito WPSS, ${ }^{19}$ que é um índice prognóstico baseado na classificação OMS, cariótipo e necessidade transfusional do paciente. ${ }^{8,9,18}$

Por isso, análise da medula óssea pela citometria de fluxo tem sido recomendada na rotina diagnóstica quando há dúvidas na citologia e o cariótipo é normal ou não fornece mitoses. ${ }^{9,18}$ É mais rápido do que a citogenética e mais barato do que o FISH. Pode também ser usado seqüencialmente no mesmo paciente para rastrear a progressão do clone anormal (número de CD34+ e aumento do número de anormalidades fenotípicas). Desta forma, a análise celular pela citometria de fluxo multiparamétrica se torna uma ferramenta útil no diagnóstico e acompanhamento das SMDs, a exemplo do que já ocorre nas síndromes linfoproliferativas $^{20}$ e nas leucemias agudas. ${ }^{21}$

No sentido de se racionalizar esta técnica poderíamos recomendar um protocolo de quatro cores usando-se as combinações:

CD11b/CD34/CD45/CD33;
HLA-DR/CD14/CD45/CD33;
CD16/CD34/CD45/CD13;
CD71/glicoforina A/CD45/CD33.
ções:

Um protocolo de três cores poderia usar as combina-

CD16/CD13/CD45 e CD16/CD11b/CD45 para granulócitos e monócitos;

HLA-DR/CD14/CD45 para monócitos;

CD71/Glicoforina A/CD45 para eritroblastos; CD15/CD34/CD45 para blastos.

A análise deve, de preferência, ser quantitativa (não só a morfologia dos plots) e deve ser sempre correlacionada com os achados microscópicos.

Para tal análise se necessita de $1 \mathrm{ml}$ de medula, que pode ser conservada à temperatura ambiente por 24 horas até o processamento, sem que haja perda de expressão antigênica. Apenas para o estudo da proliferação e apoptose, o material deve ser processado imediatamente. Isto permite que se enviem amostras para laboratórios de referência para análise.

Para a análise, em geral, devem-se analisar 30.000 células. Mas, se o objetivo é o estudo dos blastos, devem-se adquirir até 100.000 células para que a análise seja segura e reproduzível.

\section{Abstract}

The diagnosis of MDS is based on the presence of peripheral cytopenias together with cell atypias in bone marrow precursors and cytogenetic abnormalities. However, in several cases, the cell atypias are discrete, and/or the karyotype is normal, precluding a clear-cut diagnosis. Multiparametric flow cytometry is a fast, reproducible and relatively inexpensive technique, which is able to disclose changes in the expression of lineage and maturation related antigens. Several of such abnormalities have been described in MDS. Three or four-color protocols have been used to analyze erythroblasts, granulocytes, monocytes and blasts, permitting, in most of the cases, the differential diagnosis between MDS and non-clonal disorders presenting pancytopenia. Flow cytometry is also useful in the follow-up of the patients, since it has been described that progression of the MDS clone is accompanied by an increase in the number of phenotypic abnormalities and of CD34+ cells, as well as the decrease in pro-apoptotic markers in bone marrrow cells. Rev. bras. hematol. hemoter. 2006;28 (3): 178-181.

Key words: Myelodysplasia; cell maturation; antigen expression; flow cytometry.

\section{Referências Bibliográficas}

1. Lorand-Metze L, Pinheiro MP, Ribeiro E, Paula EV, Metze K. Factors influencing survival in myelodysplastic syndromes in a Brazilian population: comparison of FAB and WHO classifications. Leukemia Res 2004;28:587-594.

2. Lorand-Metze I, Meira DG, Lima CSP, Vassallo J, Metze K. The differential diagnosis between aplastic anemia and hypocellular myelodysplasia in patients with pancytopenia. Haematologica 1999; 84: 564-565

3. Magalhães SMM, Lorand-Metze I. Síndromes mielodisplásicas: protocolo de exclusão. Rev Bras Hematol Hemoter 2004; 26:263-267.

4. Magalhães SMM, Rocha Filho FD, Vassallo J, Lorand-Metze I. Nódulos linfóides medulares. Rev Bras Hematol Hemoter 2003; 25:81-87.

5. Béné MC, Feuillard J, Bernard H, Maynadié M, and the GEIL. Immunophenotyping of myelodysplasia. Clin Appl Imunol Rev 2005; 5:133-148.

6. Stetler-Stevenson M, Arthur DC, Jabbour N, Xie XY, Molldrem J, Barret $\mathrm{J}$, et al. Diagnostic utility of flow cytometry immunophenotyping in myelodysplastic syndrome. Blood 2001;98:979-987.

7. Del Cañizo MC, Fernandez E, López A, Vidriales B, Villarón E, Arroyo $\mathrm{JL}$, et al. Immunophenotypic analysis of myelodysplastic syndromes. Haematologica 2003;88:402-407.

8. Wells DA, Benesch M, Loken MR, Vallejo C, Myerson D, Leisernring WM, et al. Myeloid and monocytic dyspoiesis as determined by flow cytometry scoring in myelodysplastic syndromes correlates with the IPSS and with outcome after hemopoietic stem cell transplantation. Blood 2003;102:394-405.

9. Kussick SJ, Fromm JR, Rossini A, Ying L, Chang A, Norwood TH, et al. Four color flow cytometry shows strong concordance with bone marrow morphology and cytogenetics in the evaluation for myelodysplasia. Am J Clin Pathol 2005; 124:170-181

10. Orfao A, Ortuño F, De Santiago M, Lopez A, San Miguel JF. Immunophenotyping of acute leukemias and myelodysplastic syndromes. Cytometry 2004;58: 62-71.

11. Ogata K, Nakamura K, Yokose N, Tamura H, Tachibana M, Tanigushi $\mathrm{O}$, et al. Clinical significance of phenotypic features of blasts in patients with myelodysplastic syndromes, Blood 2002; 100:3887-3895.

12. Xu DS, Schultz C, Akker Y. Cannizzaro L, Ramesh KH, Juan DU, et al. Evidence for expression on early myeloid antigens in mature, non-blast myeloid cells in myelodysplasia. Am J Haematol 2003; 74:9-16.

13. Parker JE, Mufti GJ, Rassol F, Mijovic A, Devereux S, Pagliuca A. The role of apoptosis, proliferation and the bcl-2-related proteins in the myelodysplastic syndromes and acute myeloid leukemia secondary to MDS. Blood 2000;96:3932. 
14. Ribeiro E, Lima CSP, Metze K, Lorand-Metze I. Flow cytometry analysis of the expression of Fas/Fas-L in bone marrow CD34+ cells in myelodysplastic syndromes. Relation to disease progression. Leukemia \& Lymphoma 2004;45:309-313.

15. Ribeiro E, Matarraz Sudón S, Santiago M, Lima CSP, Metze K, Giralt M, et al. Maturation-associated immunophenotypic abnormalities in bone marrow B-lymphocytes in myelodysplastic syndromes. Leuk Res 2006; 30:9-16.

16. Van Lochem EG, Van Der Velden VHJ, Wind HK, Marvelde JG, Westerdaal NAC, Van Dongen JJM. Immunophenotypic differentiation patterns of normal hematopoiesis in human bone marrow: reference patterns for age-related changes and disease-induced shifts. Cytometry 2004;60:1-13.

17. Dunphy CH, Orton SO, Mantell J. Relative contributions of enzyme cytochemistry and flow cytometric immunophenotyping to the evaluation of acute myeloid leukemias with a monocytic component and of flow cytometric immunophenotyping to the evaluation of absolute monocytoses. Am J Clin Pathol 2004; 122: 865-874.

18. Lorand-Metze I, Ribeiro E, Lima CSP, Batista LS, Metze K. Detection of hematopoietic maturation abnormalities by flow cytometry in myelodysplastic syndromes and its utility for the differential diagnosis with nonclonal disorders. Leukemia Res, in press.

19. Malcovati L, Germing U, Kuendgen A, Della Porta MG, Invernizzi R, Giagounidis A, et al. A.. WHO classification-based prognostic scoring system (WPSS) for predicting survival in myelodysplastic syndromes. Blood 2005; 106:232a-233a.

20. Costa FPS, Pereira FG, Vassallo J, Freitas LLL, Lorand-Metze I. A utilidade da citologia por punção com agulha fina aliada a imunofenotipagem no diagnóstico dos linfomas não Hodgkin. Rev Bras Hematol Hemoter, 2005;27:16-20.

21. Lorand-Metze I. O tratamento da leucemia mielóide aguda no Brasil: o que já progredimos e o que podemos melhorar. Rev Bras Hematol Hemoter 2003; 25:1-2.

Avaliação: Editor e dois revisores externos.

Conflito de interesse: não declarado

Recebido: 09/03/2006

Aceito após modificações: 30/03/2006 